\title{
Ultrastructural Localization of Slow Retrograde Axonal Transport: An Autoradiographic Study ${ }^{1}$
}

\author{
MARINA MATA, ${ }^{2}$ JULIE STAPLE, AND DAVID J. FINK \\ Neurology Research Laboratory, University of Michigan and Veterans Administration Medical Center, Ann Arbor, Michigan 48105
}

\begin{abstract}
We used $\left[{ }^{3} \mathrm{H}\right]-\mathrm{N}$-succinimidylpropionate $\left(\left[{ }^{3} \mathrm{H}\right]-\mathrm{N}-\mathrm{SP}\right)$ to covalently label endogenous intra-axonal proteins within the nerve in order to study their bidirectional transport. At the time of injection virtually all of the labeled proteins are found at the injection site. At later times specific patterns of labeled proteins are found within the nerve both proximal to and distal from the injection site, as a result of retrograde and anterograde axonal transport, respectively. We undertook the current study to determine the ultrastructural distribution of the $\left[{ }^{3} \mathrm{H}\right]-\mathrm{N}$-SP-labeled transported proteins in the nerve.

One microliter of $\left[{ }^{3} \mathrm{H}\right]-\mathrm{N}$-SP was injected subepineurially in sciatic nerve, and 5 days later the nerves were processed either for light and electron microscopic autoradiography or for gel electrophoresis and fluorography. At the injection site the labeled proteins are predominantly myelin proteins. Distally, a pattern similar to that described for slow anterograde transport is seen. Proximal to the injection site a constellation dominated by the 68-kilodalton protein is seen.

Light microscopic autoradiography shows diffuse labeling both in axons and in myelin at the injection site, with predominant axonal labeling distant from the injection site. Electron microscopic autoradiography of segments distal to the injection site show silver grains which are distributed within the axoplasm without apparent relationship to organelles. In contrast, segments proximal to the injection site show silver grains which seem closely related to membrane-bound organelles, predominantly mitochondria.

These results suggest that slow retrograde transport has a unique subcellular distribution that is distinct from that of slow anterograde transport.
\end{abstract}

We have previously used the radioactive acylating agent $\left[{ }^{3} \mathrm{H}\right]-\mathrm{N}$ succinimidylpropionate $\left(\left[{ }^{3} \mathrm{H}\right]-\mathrm{N}-\mathrm{SP}\right)$ to covalently label endogenous axonal proteins in order to study their subsequent bidirectional transport. We have used the method to investigate transport in the hypothalamo-pituitary axis (Fink and Gainer, 1979) and the rat sciatic nerve (Fink and Gainer, 1980a, b), and others have applied it to the goldfish optic tract (Williams and Agranoff, 1983) and the Xenopus optic tract (Szaro et al., 1981). In addition, [ $\left.{ }^{3} \mathrm{H}\right]-\mathrm{N}$-SP has been used

Received January 7, 1985; Revised March 22, 1985;

Accepted April 10, 1985

${ }^{1}$ This work was supported by Veterans Administration Merit Review grants Io M. M. and D. J. F. We wish lo thank Di. Tom Morrow for assistance with the statistical analysis

${ }^{2}$ To whom correspondence should be addressed, at Neurology Service (127), Veterans Administration Medical Center, 2215 Fuller Road, Ann Arbor, Michigan 48105. as an anatomic tracer of fibers in passage (Hedreen and Holm 1981), and the iodinated analogue Bolton-Hunter reagent has been used as a vital stain in developmental studies (Katz et al., 1982).

In the rat sciatic nerve, intraneural injection of $\left.{ }^{3} \mathrm{H}\right]-\mathrm{N}$-SP labeled a constellation of proteins moving at an apparent slow rate of transport toward the nerve terminal that is similar in molecular weight to the described slow anterograde transport, and it identified, in addition, a constellation of proteins, moving back toward the cell body at an apparent rate of 3 to $5 \mathrm{~mm} /$ day, which consisted predominantly of a 68-kilodalton (kD) protein and several other bands (Fink and Gainer, 1981). The 68-kD protein appears by two-dimensional gel electrophoresis and immunoprecipitation to be similar to rat serum albumin. The origin, localization, role, and fate of the slowly retrogradely transported intra-axonal proteins are not known.

The major rate components of anterograde transport are generally accepted to be related to particular cytological components within the axon. Rapidly transported components are "particulate," have the hydrodynamic properties of membrane, and they appear to correspond to the tubulovesicular compartment of the axon. The more slowly transported components are "soluble" and correspond to the axonal cytoskeleton consisting of filaments, tubules, and the "axoplasmic matrix" (Lorenz and Willard; 1978, Lasek et al., 1984). Rapid retrograde transport appears to consist primarily of membrane-bound structures, multivesicular bodies, dense vesicles, and mitochondria, as well as membranous tubules distinct from those involved in rapid anterograde transport

We undertook the current study to define the ultrastructural distribution of the slowly retrogradely transported, labeled axonal proteins in order to determine whether slow retrograde axonal transport was associated with the axoplasmic matrix or whether the label was associated with particular subcellular structures. In this paper we describe the ultrastructural localization of intra-axonal proteins, covalently labeled with [ $\left.{ }^{3} \mathrm{H}\right]-\mathrm{N}-\mathrm{SP}$, moving bidirectionally within the axon within the time frame of slow transport. Five days after the subepineurial injection of $\left[{ }^{3} \mathrm{H}\right]-\mathrm{N}$-SP into one sciatic nerve, both sciatic nerves, the lumbosacral spinal cord, and dorsal root ganglia were processed for light microscopic (LM) and electron microscopic (EM) autoradiography. We found that anterogradely transported, labeled proteins appear to be distributed diffusely through the axoplasmic matrix, in agreement with the findings employing trace labeling at the cell body, whereas slowly retrogradely transported, labeled proteins appear in close association with organelles.

\section{Materials and Methods}

Iniections. Male Spraque-Dawley rats (250 to $400 \mathrm{gm}$ ) were used in all experiments. $\left.{ }^{3} \mathrm{H}\right]-\mathrm{N}$-SP (Amersham; specific activity, $50 \mathrm{Ci} / \mathrm{mmol}$ ) was injected subepineurially as described previously (Fink and Gainer,' 1980). Briefly, the $\left[{ }^{3} \mathrm{H}\right]-N$-SP was dried under a stream of nitrogen and resuspended in saline. One microliter containing 40 or $80 \mu \mathrm{Ci}$ of $\left[{ }^{3} \mathrm{H}\right]-N$-SP was injected over 5 min using a 30 gauge needle attached to a Hamilton syringe in a 
Harvard infusion pump. The wound was closed with wound clips, and 5 days after injection the animals were sacrificed and the neves were processed for biochemical or morphologic studies.

Biochemical procedures. The animals were sacrificed by decapitation. The sciatic nerves were removed en bloc and frozen rapidly on dry ice. The nerves were cut into 3-mm segments and homogenized in $1 \mathrm{ml}$ of $5 \mathrm{~mm}$ Tris, $5 \mathrm{~mm} \mathrm{NaCl}, \mathrm{pH}$ 7.4. Aliquots were removed for determination of trichloroacetic acid (TCA)-precipitable radioactivity by conventional filter pad procedures. The samples of interest were precipitated twice with 10\% TCA and washed sequentially with acetone and ether; then, the pellet was suspended in a small volume of $0.0625 \mathrm{M}$ Tris, $2 \%$ (SDS), $5 \%$ beta mercapto ethanol (BME), and $8 \mathrm{M}$ urea. Samples roughly equalized for total disintegrations per minute were run on a 5 to $15 \%$ gradient polyacrylamide slab gel, using the Laemiril buffer system (Laemmli, 1970). The gels were dried, processed for autoradiography using EnHance (New England Nuclear), and developed on Kodak X-AR film.

Light and electron microscopic autoradiography. Four animals were processed for autoradiography. Under chloral hydrate anesthesia the animals were perfused through the heart with $0.1 \mathrm{M}$ phosphate buffer followed by $3 \%$ glutaraldehyde, $0.5 \%$ paraformaldehyde in $0.1 \mathrm{M}$ phosphate buffer, $\mathrm{pH}$ 7.4, for approximately $1 \mathrm{hr}$. Sciatic nevves, lumbosacral spinal cord, and dorsal root ganglia were dissected out and kept in fixative for an additional $2 \mathrm{hr}$ at $4^{\circ} \mathrm{C}$. The tissue was washed overnight in phosphate buffer at $4^{\circ} \mathrm{C}$ The nervos and spinal cords were then cut into consecutive $2-\mathrm{mm}$ segments, and the tissue was postfixed in $2 \% \mathrm{OsO}_{4}$ in the same buffer, dehydrated in ethanol, and embedded in Polybed-Araldite mixture. For LM autoradiography, semilhin $(1 \mu \mathrm{m})$ sections were coated with Kodak NTB-2 nuclear tract emulsion. After exposure in the dark at $4^{\circ} \mathrm{C}$ for 1 day to 7 weeks, sections were developed in Kodak D-19 developer and mounted in Permount. For EM autoradiography ultrathın sections $(50 \mathrm{~nm})$ were placed on glass slides coated with $1 \%$ parlodion. The slides were coated by the dipping technique with llford-4 nuclear tract emulsion. After exposure in the dark at $4^{\circ} \mathrm{C}$ for 3 days to 24 weeks, the sections were developed in Kodak D-19 developer and transferred to 300 mesh nickel grids. Sections stained with uranyl acetate were examined in a JEOL 100 electron microscope.

The EM autoradiographs were analyzed using the method described by Whur et al. (1969). Concentric circles of increasing radius were drawn on a clear plastic sheet used as a stencil, their size being proportional to the magnification and to the approximate maximum distance $(225 \mathrm{~nm})$ that the silver grain travels from the radioactive source in our conditions. This stencil was placed over pictures of radioautographs so that each silver grain was placed centrally within a circle. The structures within the circle were identified and given one point. The number of points received by any given structure was expressed as the percentage of the total number of grains counted.

The EM autoradiographs of the sciatic nerve were analyzed for the number of silver grains in axons in each of the following compartments: mitochondria smooth endoplasmic reticulum (SER), tubulovesicular profiles, other organelles (multivesicular bodies and large dense bodies) and axoplasm. A total of 264 axons containing 1750 silver grains were analyzed. Approximately half the grains were from nerve segments proximal to the injection site and the other half were from nerve segments distal to the injection site.

\section{Results}

Gel electrophoresis of the segments of nerve $4 \frac{1}{2}$ days after injection of $\left[{ }^{3} \mathrm{H}\right]-N-S P$ is seen in Figure 1 . The injection site is dominated by a labeled protein band of apparent $M_{r}=27,000$, which is similar in size to the $P_{0}$ protein, the major protein constituent of myelin. Anterograde from the injection site, the first three 3-mm segments (up to $9 \mathrm{~mm}$ from the injection site and, therefore, representing a transport rate of up to $2 \mathrm{~mm} /$ day), are dominated by a triplet of labeled proteins of apparent $M_{r}=200,000,150,000$, and 69,000 , as well as a less heavily labeled doublet in the $53-$ to $55-\mathrm{kD}$ range. These proteins are similar in molecular weight distribution to the neurofilament triplet and tubulin which are known to be transported in the sciatic nerve at slow rates. The segments more distal from the injection site contain a more complex pattern of proteins and the higher molecular weight components (200 and $150 \mathrm{kD}$ ) are not seen.

Proximal to the injection site, proteins which are transported retrogradely back toward the cell body are labeled. In all of the 3 $\mathrm{mm}$ segments (apparent transport rate up to $5 \mathrm{~mm} /$ day), the pattern is similar, dominated by a $68 \mathrm{kD}$ band, with other components of $M_{r}$ $=72,000,50,000,42,000$, and 25,000.
The distribution of the labeled proteins was confirmed using LM autoradiography. At the injection site 5 days after the injection of $\left[{ }^{3} \mathrm{H}\right]-N-S P$, the main nerve fascicle is heavily labeled with silver grains concentrated in the epineurium, perineurium, and myelin. The axoplasm is relatively unlabeled, giving the nerve fibers the appearance of black rings around clear centers (Fig. $2 A$ ). Twelve to $16 \mathrm{~mm}$ distal trom the injection site (representing anterograde transport), the axons are preferentially labeled, and relatively fewer grains are found in the myelin, epineurium, and perineurium. The myelinated axons have an appearance inverse to that found at the injection site, with a dark center and a clear surround (Fig. $2 B$ ).

Twelve to $16 \mathrm{~mm}$ proximal to the injection site (representing retrograde transport) there is also preferential labeling of axons, with grains clustered in those axons which have been labeled (Fig. ?C) In agreement with the biochemical data, the grain density in the retrograde direction is lower than that found in segments a similar distance in the anterograde direction from the injection site. The extra-axonal grains are more apparent in these Polybed-Aralditeembedded sections than they were in the previous paraffin-embedded sections (Fink and Gainer, 1980) in accordance with the better preservation generally of endoneurial substance. They were most prominent at the injection site, and their densily fell off syrmirletrically in both directions as the distance from the injection site increased. They appeared to be preferentially distributed in the endoneurial perivascular space and in the epineurium.

The cell bodies giving rise to axons in the sciatic nerve found in the lumbosacral spinal cord (motor) and lower lumbar dorsal root ganglia (sensory). At 5 days after $\left[{ }^{3} \mathrm{H}\right]-N$-SP injection silver grains were found over several large neuronal cell bodies of the ipsilateral anterior horn but were not found over the contralateral anterior horn. The grains were localized over the cytoplasm and the initial segment of the axon but spared the nucleus. A rim of grains was seen around the neuronal cell bodies, suggesting labeling of the dendritic tree (Fig. 2E). LM autoradiography of the corresponding lumbosacral dorsal root ganglia revealed selective accumulation of silver grains within the cytoplasm of the neurons and within some axons (Fig. $2 D$ ), with extrcmoly low labeling in the endoneurium and capsule and virtually no grains seen in the contralateral dorsal root ganglia. In general, the amount of labeling in dorsal root ganglia cells appeared greater than that in spinal cord neurons.

Blocks selected from the LM autoradiography were sectioned for EM autoradiography. Analysis of the intra-axonal grains 12 to $16 \mathrm{~mm}$ distal from the injection site showed multiple silver grains randomly distributed through the axoplasm. The labeled proteins are found over the axoplasmic matrix, predominantly in regions devoid of organelles, and uncommonly associated with any organelles (Fig. 3). EM autoradiograms of sections obtained 12 to $16 \mathrm{~mm}$ proximal to the injection site showed few silver grains free in the axoplasm. Most were closely associated with the various organelles in the axon, particularly mitochondria, whereas fewer were tound associated with SER, tubulovesicular profiles, and multivesicular bodies (Fig. 4). Grains were often seen in organelles clustered in the paranodal region at nodes of Ranvier (Fig., 5, $A$ and $B$ ) and were found associated with organelles in unmyelinated as well as myelinated fibers (Fig. 5C).

For quantitative analysis of grain distribution 12 randomly selected grid squares were analyzed. The 8 grid squares of nerve proximal to the injection site had been developed 5 to 24 weeks. The 4 grid squares of nerve distal to the injection site had been developed 3 to 5 days. Of the 1797 grains in the grids of proximal nerve, $55 \%$ appeared within the axoplasm, $15 \%$ in myelin, $7 \%$ in Schwann cell cytoplasm, and $26 \%$ was endoneurial. Of the 2672 grains in the distal grids, $86 \%$ were within the axoplasm, $5 \%$ in myelin, $2 \%$ in Schwann cells, and $7 \%$ was endoneurial. The ultrastructural distribution of intra-axonal grains was determined using the method of Whur et al. (1969), and the results are shown in Table I. The quantitative analysis confirms that, distally, grains appear significantly more frequently free in the axoplasm and, proximally, they 
Fig. 1. Fluorograph of SDS gradient gel electrophoresis of labeled proteins, $41 / 2$ days after injection of $\left[{ }^{3} \mathrm{H}\right]-\mathrm{N}$ SP. Each well represents a consecutive 3-mm segment of nerve, from which aliquots roughly equalized for TCAprecipitable disintegrations per minute were applied. At the injection site (large arrowhead) principally myelin protein remains labeled. In the anterograde direction toward the nerve terminal (to the right of the injection site) patterns similar to slow transport are seen. In the retrograde direction (to the left of the injection site on the gel) toward the cell body the patterns are dominated by a $68-\mathrm{kD}$ peak with smaller components of $70,50,42$, and $25 \mathrm{kD}$. The molecular weight standards (small arrowheads) are $200,000,92,000,68,000,45,000$, and 31,000.

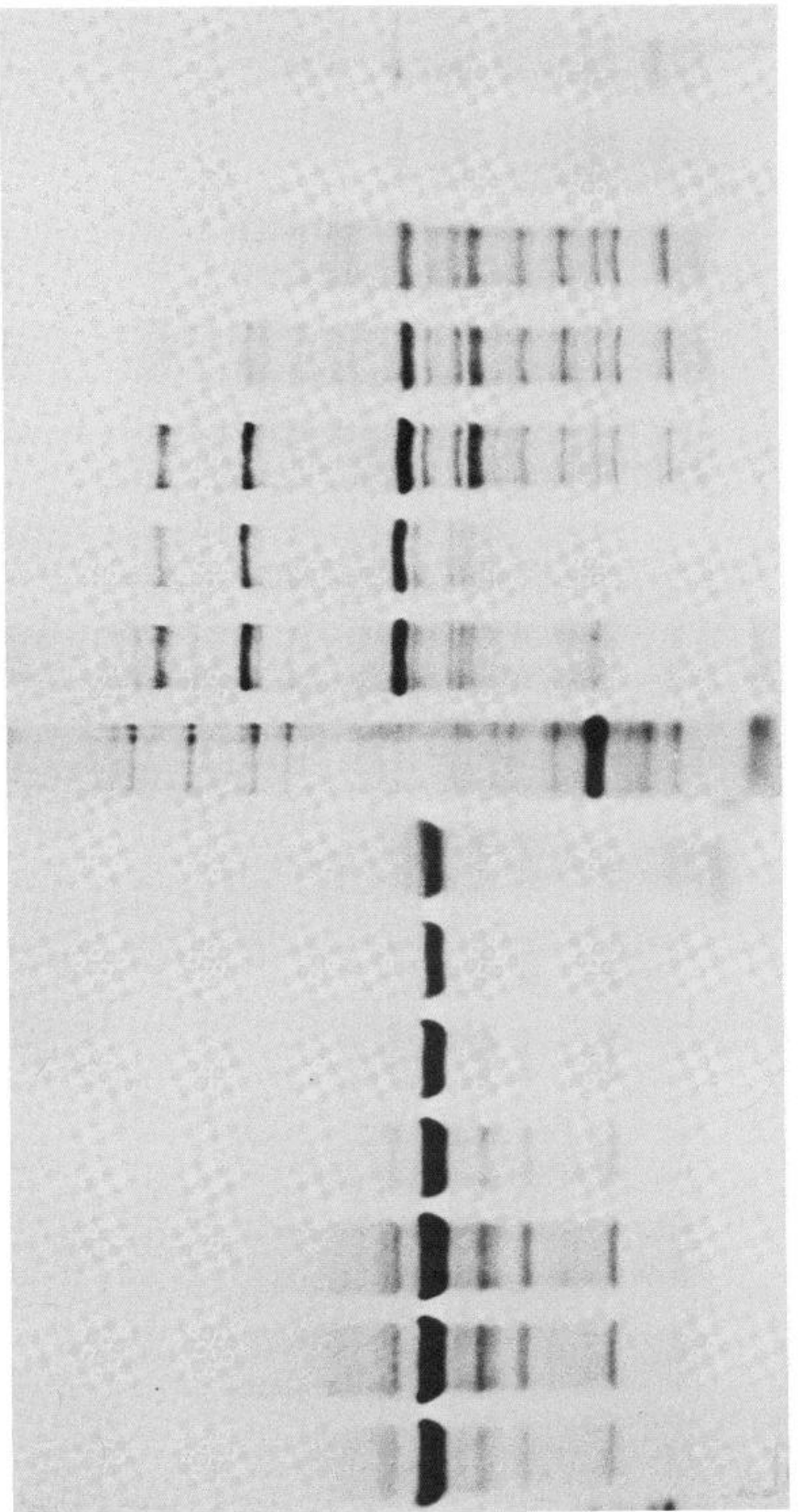

appear significantly more frequently associated with mitochondria. The apparent association of anterogradely transported label with SER may reflect the high density of axoplasmic labeling.

\section{Discussion}

The results of this study confirm and extend previous data regarding anterograde axonal transport and offer new insight into the phenomenon of retrograde axonal transport. Biochemical studies utilizing the injection of radioactive amino acids into the region of the cell body have shown that slow anterograde transport consists of the constituents of the axoplasmic matrix and the axonal cytoskeleton (Hoffman and Lasek, 1975; Lasek et al., 1984). Similarly, 5 days after an intraneural injection of $\left[{ }^{3} \mathrm{H}\right]-\mathrm{N}$-SP those labeled proteins seen up to $9 \mathrm{~mm}$ from the injection site are similar in molecular weight to the neurofilament triplet and tubulin.

There are two previous studies of the ultrastructural distribution of slowly transported proteins using autoradiography. Droz et al. (1973) 


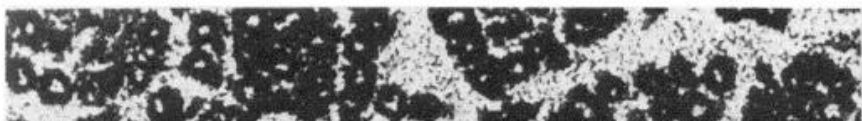
6.5. 4.

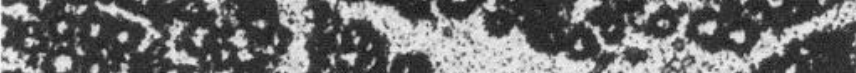
1.

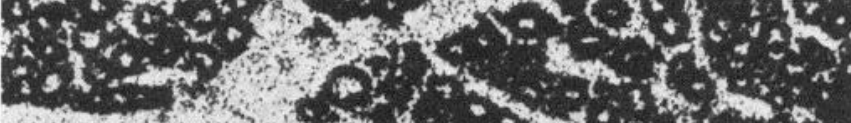

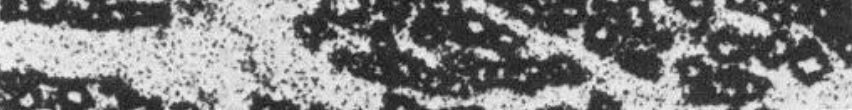

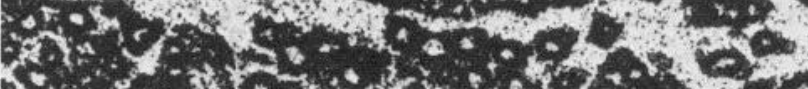

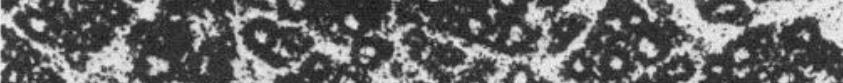

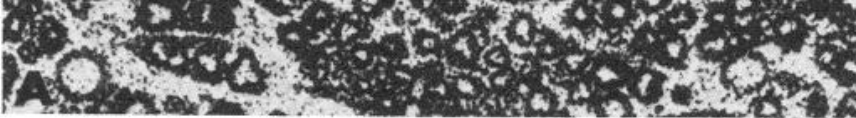

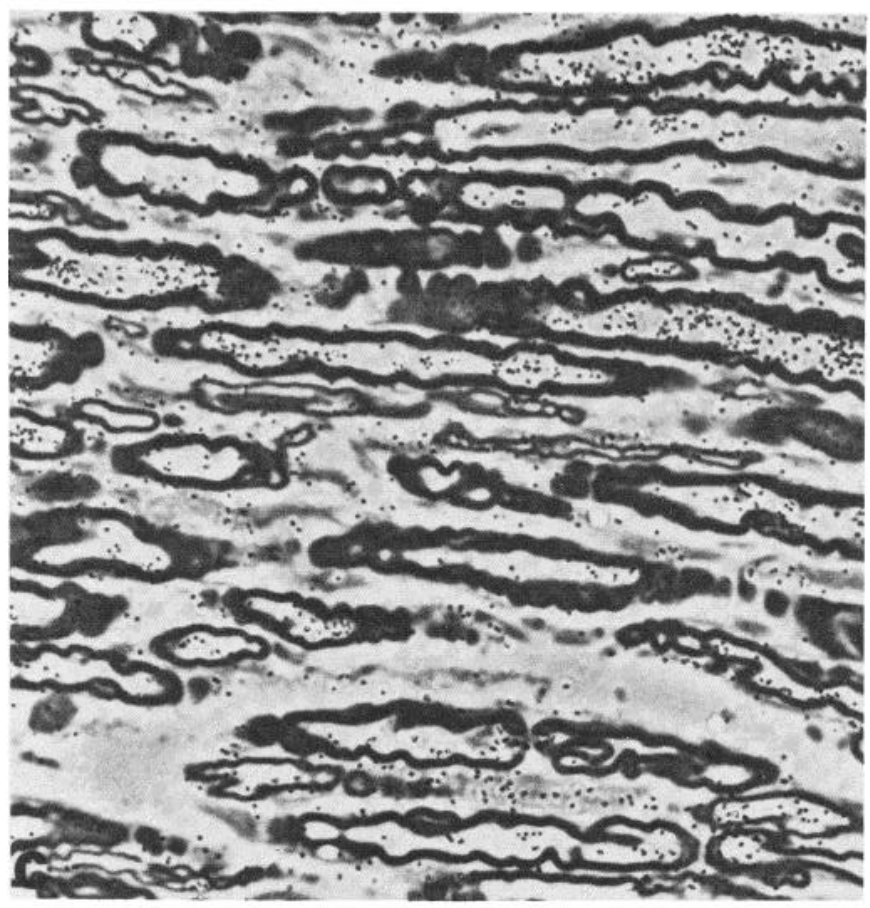

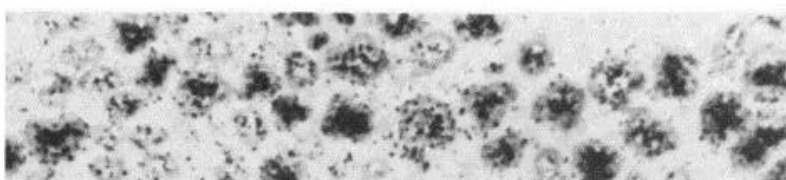

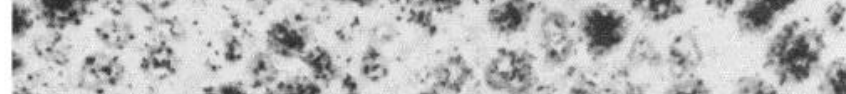

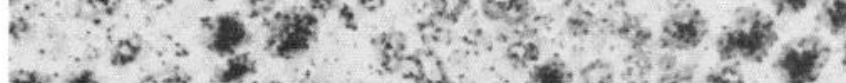
$4+4$ को 4 b.

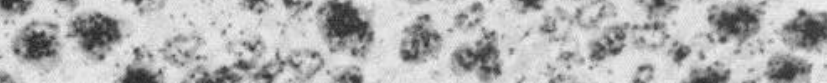

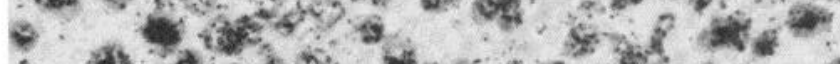

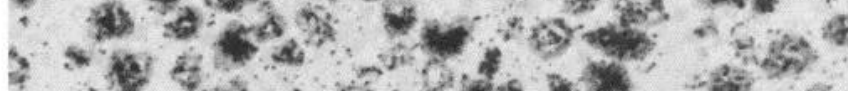

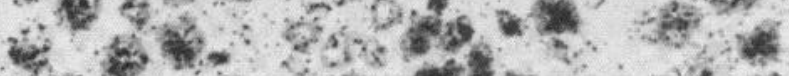

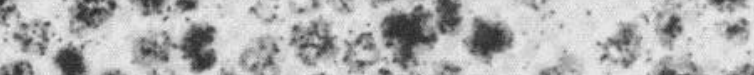

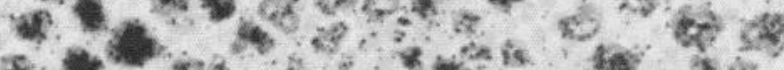

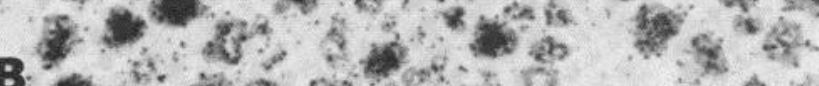

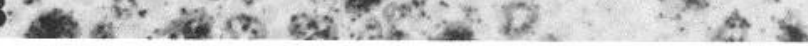

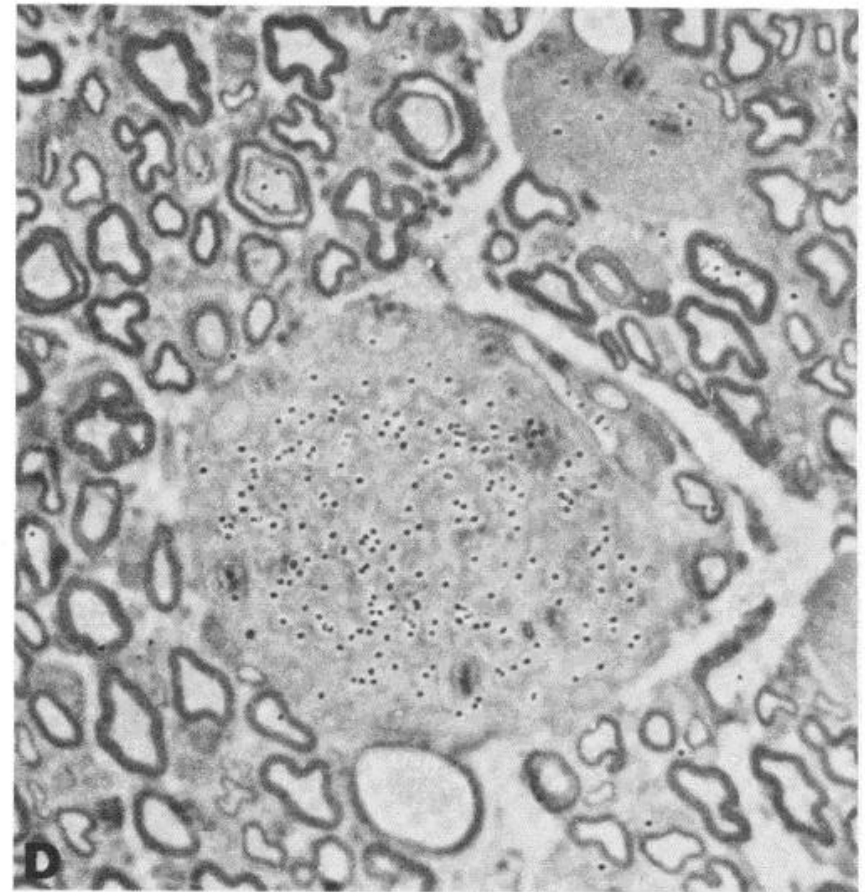

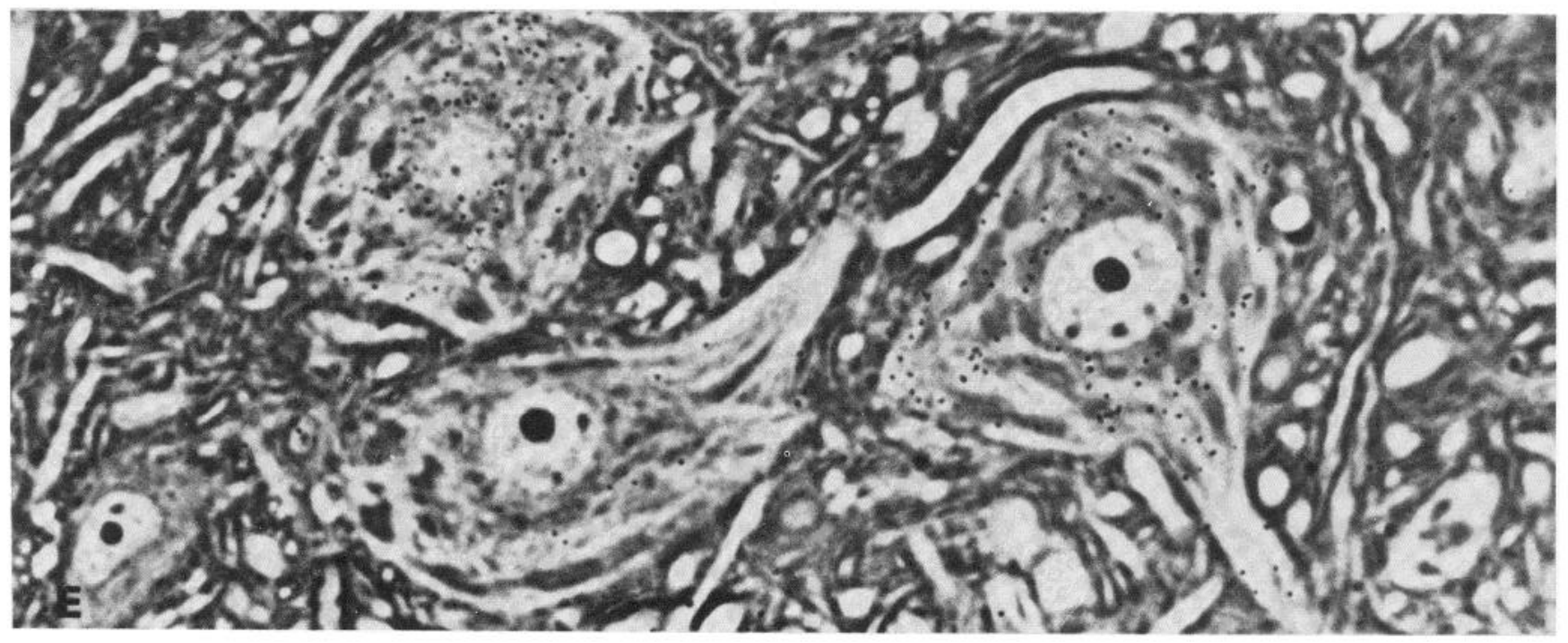

Figure 2. LM autoradiography 5 days after injection of $\left[{ }^{3} \mathrm{H}\right]-\mathrm{N}-\mathrm{SP}$ into sciatic nerve. At the injection site $(A)$, myelin is predominantly labeled. Twelve to 16 $\mathrm{mm}$ distal from the injection site $(B)$, there is preferential labeling of axons. Twleve to $16 \mathrm{~mm}$ proximal to the injection site $(C)$, axons are preferentially labeled, although there are fewer grains than in the anterograde direction. Ipsilateral lumbar dorsal root ganglion cells $(D)$ and lumbar spinal cord ventral horn cells $(E)$ are also selectively labeled. Magnifications and exposures: $A, \times 980,1$ day; $B, \times 990,2$ weeks; $C, \times 800,2$ weeks; $D, \times 880,3$ weeks; $E$, 

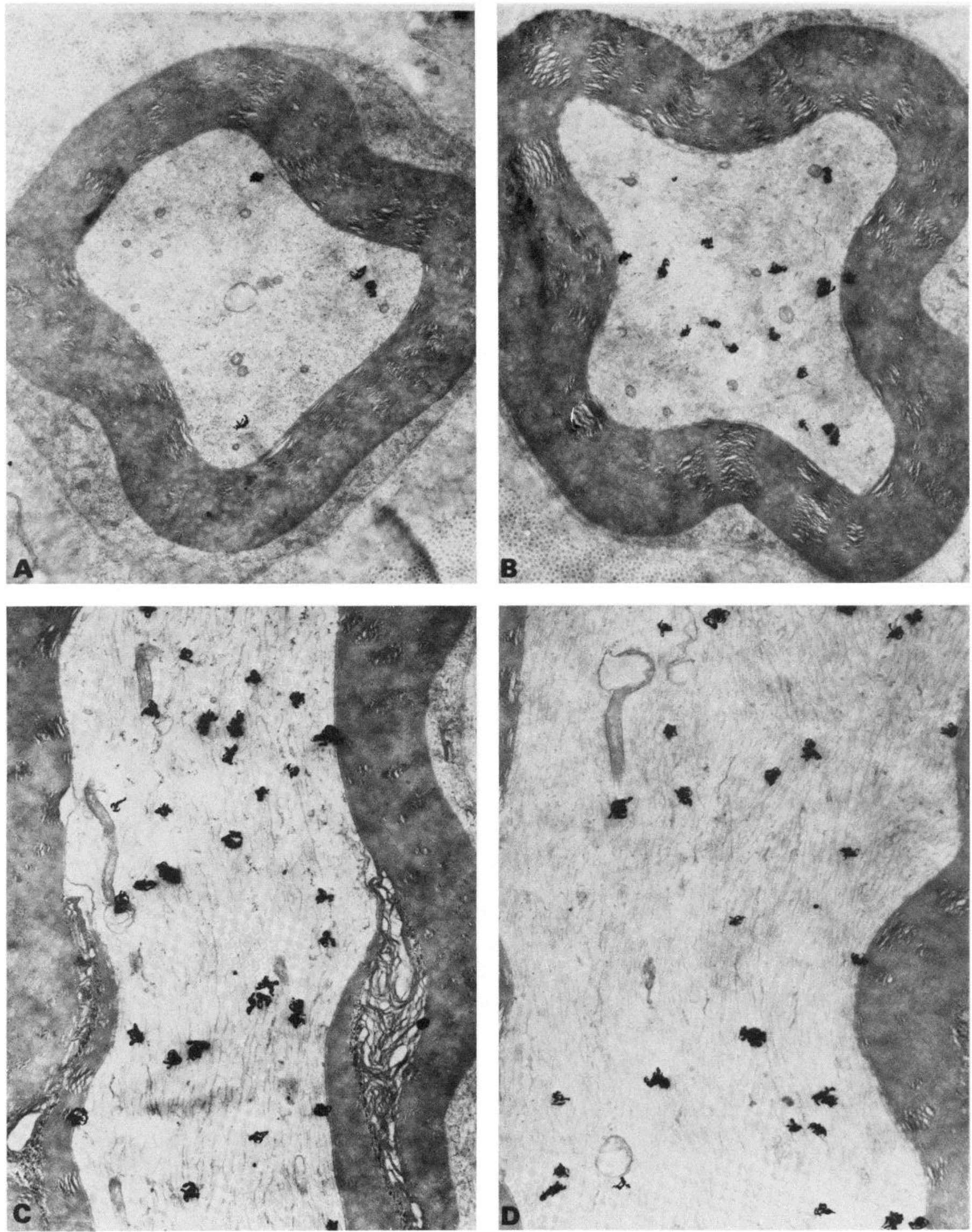

Figure 3. EM autoradiography of a sciatic nerve, 12 to $16 \mathrm{~mm}$ distal from the injection site (anterograde transport). Silver grains are scattered throughout the axoplasm, predominantly in areas of axoplasmic matrix. Magnifications: $A, \times 13,600 ; B, \times 13,600 ; C, \times 18,000 ; D, \times 18,000$; all at 4 days exposure. 

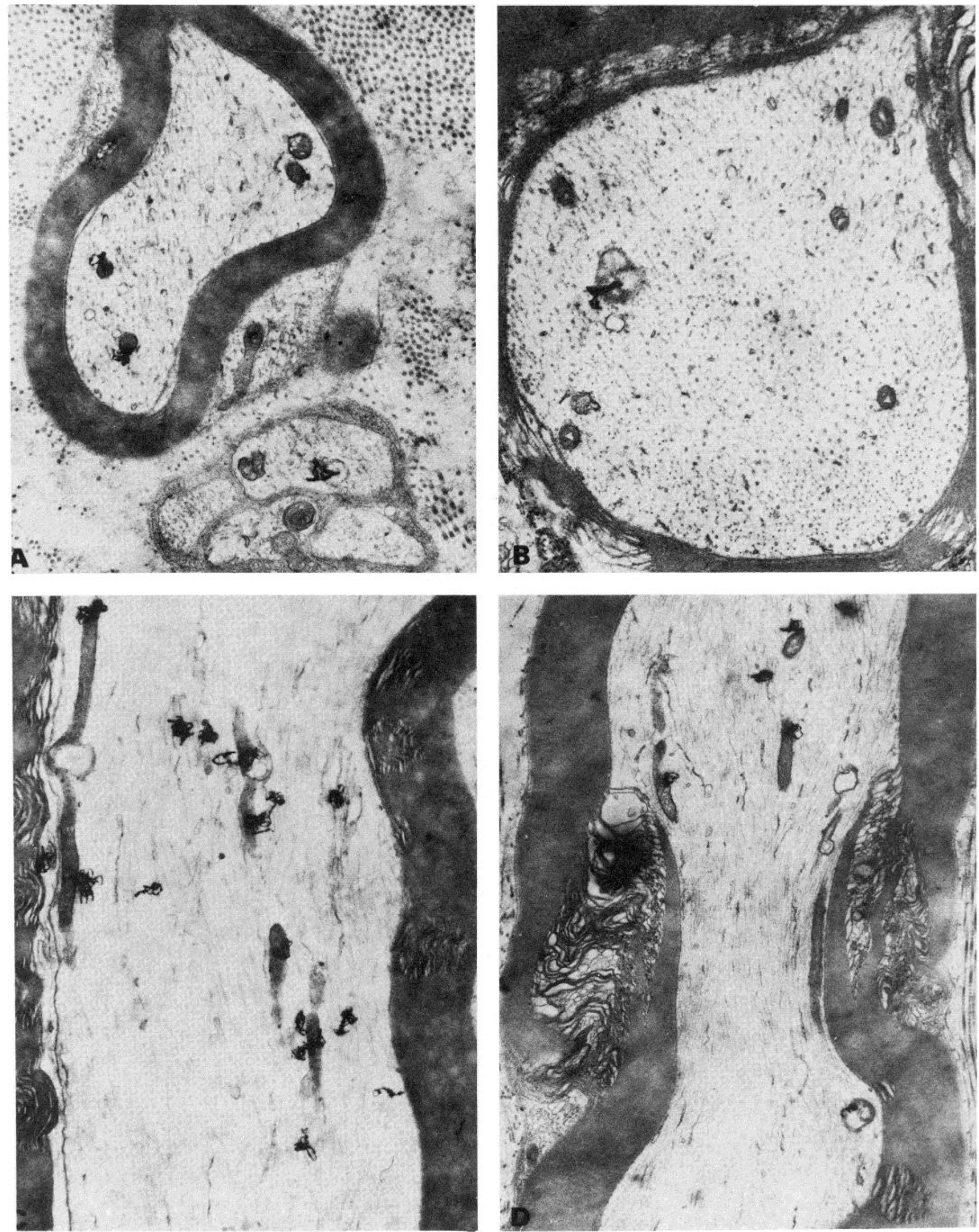

Figure 4. EM autoradiography of sciatic nerve 12 to $16 \mathrm{~mm}$ proximal to the injection site (retrograde transport). Silver grains are found in close relationship to organelles. Mitochondria, less commonly, tubulovesicular profiles, SER, and multivesicular bodies are all labeled. Few grains are seen free in the cytoplasmic matrix. Magnifications: $A, \times 21,000 ; B, \times 31,500 ; C, \times 19,000 ; D, \times 17,600$; all at 7 weeks exposure. 

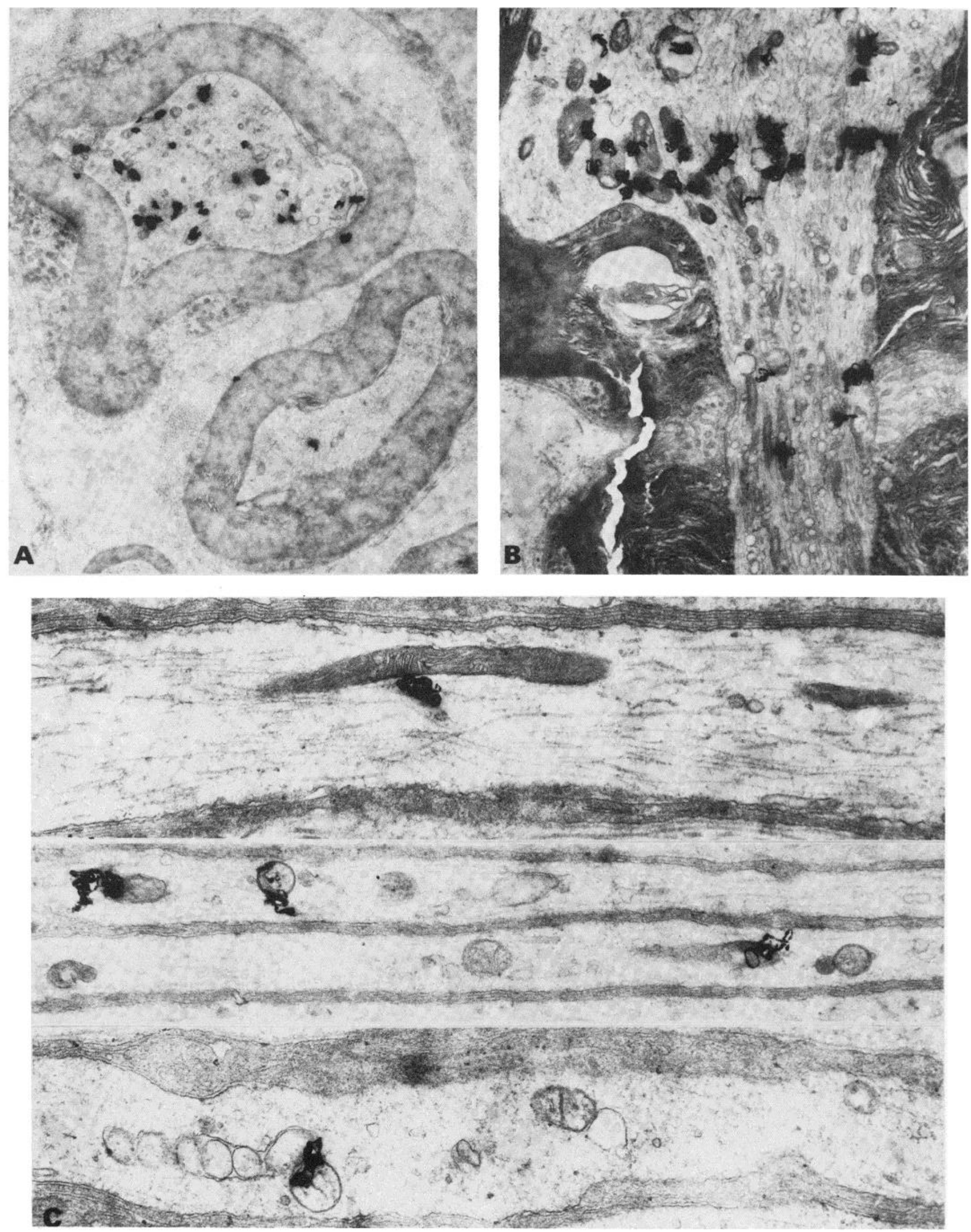

Figure 5. EM autoradiography of sciatic nerve, 12 to $16 \mathrm{~mm}$ proximal to the injection site. At nodes of Ranvier and in paranodal regions $(A$ and $B)$ where there typically are dense accumulations of organelles, radioactive labeling was particularly prominent. Labeling of organelles occurred in unmyelinated fibers (C) as well as in myelinated fibers. Magnifications and exposures: $A, \times 8,750,7$ weeks; $B, \times 17,600,4$ weeks; $C$, top, $\times 36,000,4$ weeks; middle, $\times$ $31,200,7$ weeks; bottom, $\times 37,500,7$ weeks. 
TABLE I

Distribution of intra-axonal siver grains

The ultrastructural distribution of intra-axonal silver grains was determined by the method of Whur et al. (1969). A total of 264 axons were counted. Probability $(p)$ values were calculated using the $\chi^{2}$ test for two independent variables.

\begin{tabular}{lrrrrrl}
\hline & \multicolumn{2}{c}{ Distal } & & \multicolumn{2}{c}{ Proximal } & \multirow{2}{*}{ Probability } \\
\cline { 2 - 3 } & Number & $\%$ & & Number & $\%$ & \\
\hline Mitochondria & 106 & 11 & & 559 & 70 & $<0.001$ \\
SER & 166 & 18 & 51 & 6 & $<0.001$ \\
Tubulovesicular & 63 & 7 & 61 & 8 & \\
Other & 1 & $<1$ & 6 & $<1$ & \\
Axoplasm & 603 & 64 & 125 & 15 & $<0.001$ \\
Total & 938 & & 812 & & \\
\hline
\end{tabular}

showed that, following the intracerebral injection of $\left[{ }^{3} \mathrm{H}\right]$ lysine in chicks, radioactive grains accumulating in the preganglionic axons and terminals of the ciliary ganglion were found in regions of filaments and microtubules and, to a lesser extent, over mitochondria, in contrast to rapidly transported label which appeared to be associated predominantly with membranous profiles. Similarly, Schonbach et al. (1973) found that the slowly advancing wave of radioactivity from pigeon retina to optic tectum following the intraocular injection of [ ${ }^{3} \mathrm{H}$ leucine was localized over axoplasm, labeling microtubules, neurofilaments, and mitochondria, in contrast to their studies of rapid transport in the same system (Schonbach et al., 1971), which showed labeling of membranous profiles.

There are no previous reports of autoradiographic studies of slow anterograde transport in mammalian peripheral nerve, but our finding that the slowly anterogradely transported proteins are localized over areas of axoplasmic matrix relatively devoid or organelles and with the silver grains in no particular relationship to organelles is in agreement with the autoradiographic studies of central nervous system axons and with the biochemical identity of the slowly transported components. The region analyzed represents an overlap between the leading edge of slow component a (SCa), containing neurofilaments and tubulin, and the trailing edge of slow component $b$ (SCb), which consists of the other slowly transported proteins of the cytomatrix (Lasek et al., 1984).

In contrast to labeled slow anterograde transport, labeled proteins which have moved 12 to $16 \mathrm{~mm}$ back toward the cell body from the injection site in 5 days appear to be associated with organelles. Predominantly, mitochondria and, less commonly, SER and other vesicular profiles were labeled, but we found that areas of axoplasm devoid of organelles were rarely labeled. Unlike slow anterograde transport, then, slow retrograde transport appears to be particulate in nature.

Previous studies of relrograde transport, using other techniques, have focused on rapid retrograde transport. Direct visualization of organelles within axons using either Nomarski optics (Forman et al.. 1977) or video enhanced techniques (Allen et al., 1982; Brady, 1984) has shown that a wide variety of organelles move rapidly in both directions within the axoplasm. In the anterograde direction the majority of particles are small (100 nm or less), whereas in the retrograde direction larger particles and mitochondria predominate (Brady, 1984). Over the time course of these studies $90 \%$ of the mitochondria appears stationary within the axon (Forman et al., 1977).

The nature of the different organelles moving in each direction has been confirmed by electron microscopic analysis of organelles accumulating at a crush in Xenopus axons (Smith, 1980) or at a focal cooling in mouse saphenous nerve (Tsukita and Ishikawa, 1980) and squid giant axon (Brady, 1984). Distal to the lesion, representing rapid retrograde transport, dense lamellated bodies, multivesicular bodies, mitochondria, and a variety of other mem- brane-bound structures accumulate, whereas the accumulation proximal to the lesion consists primarily of smaller vesicles and tubulovesicular elements. Brady (1984) reports that mitochondria may be more common proximal to the lesion than distal to the lesion. The time course of these lesions studies, although longer than the studies using direct visualization, nonetheless is such that it would allow for the accumulation of rapidly but not slowly transported organelles. A third method which has been used to study retrograde transport involves the injection of macromolecular tracers in the region of nerve terminals and the subsequent ultrastructural localization of the tracer within the axon. Using horseradish peroxidase (HRP) (LaVail and LaVail, 1974; LaVail et al., 1980), it can be shown that the tracer is found within tubular structures which are distinct from the endoplasmic reticulum and also within multivesicular bodies. Whereas most of the studies using exogenous tracers have been performed over the time course of rapid transport, Corvaja et al. (1977) have demonstrated a slower peak of retrograde transport of HRP in frogs, estimated at $6 \mathrm{~mm} /$ day. In addition, following the injection of lead into mouse gastrocnemius muscle, a wave of slowly transported lead moving retrogradely in the sciatic nerve at 3 to $5 \mathrm{~mm} /$ day has been identified (Baruah et al., 1981).

The distribution of slowly retrogradely transported label which we found in this study is, like rapid retrograde transport studied by other techniques, confined to organelles. There may perhaps be a greater predominance of labeled mitochondria in slow than in rapid retrograde transport. The fact that the ultrastructural distribution of slowly retrogradely transported label is distinct from the ultrastructural distribution of slowly anterogradely transported label is further evidence that the movement of $\left[{ }^{3} \mathrm{H}\right]-\mathrm{N}$-SP-labeled proteins from the injection site represents a specific biologic process. The results suggest that, unlike anterograde transport, where the different velocities of transport appear to represent different subcellular structures, both rapid and slow retrograde transports appear to consist of a similar group of organelles, moving at different average velocities. Whether there are other biochemical differences between the organelles which are transported slowly and those which are transported rapidly remains to be determined.

We cannot say that the 68-kD peak which has tentatively been identified as serum albumin is found associated with these organelles. The $68-\mathrm{kD}$ labeled peak is predominant in the gel pattern of retrogradely transported label, and ultrastructurally, we have shown that intra-axonal retrogradely transported label is found predominantly associated with organelles. However, ultimately, the localization must rest on immunocytochemical evidence. LM immunocytochemistry shows deposition of albumin in the endoneurial space (Gold et al., 1984; M. Mata and D. J. Fink, unpublished data) and within anterior horn cells of the spinal cord (Sparrow, 1981). The determination of the distribution of intra-axonal albumin using EM immunocytochemistry is currently underway in our laboratory.

\section{References}

Allen, R. D., J. Metuzals, I. Tasaki, S. T. Brady, and S. P. Gilbert (1982) Fast axonal transport in squid giant axon. Science 218: 1127-1129.

Baruah, J. K., C. G. Rasool, W. G. Bradley, and T. L. Musat (1981) Retrograde axonal transport of lead in rat sciatic nerve. Neurology 31: 612-616.

Brady, S. T. (1984) Basic properties of fast axonal transport and the role of fast axonal transport in axonal growth. In Axonal Transport in Neuronal Growth and Regeneration, J. Elam and P. Cancalon, eds., pp. 13-30, Plenum Press, New York.

Corvaja, N., P. D'Ascanio, M. Merli, and C. Zucconi (1977) Slow rate transport of horseradish peroxidase in toads. Brain Res. 128: 537-541.

Droz, B., H. L. Koenig, and L. D. Giamberardino (1973) Axonal migration of protein and glycoprotein to nerve endings. I. Radioautographic analysis of the renewal of protein in nerve endings of chicken ciliary ganglion after intracerebral injection of $\left({ }^{3} \mathrm{H}\right)$ lysine. Brain Res. 60: 93-127.

Fink, D. J., and H. Gainer (1979) The use of labeled acylating probe for the study of fast axonal transport, in vivo. Brain Res. 177: 208-213.

Fink, D. J., and H. Gainer (1980a) Retrograde axonal transport of endogenous 
proteins in sciatic nerve demonstrated by covalent labeling in vivo. Science 208: 303-305

Fink, D. J., and H. Gainer (1980b) Axonal transport of proteins. A new view using in vivo covalent labeling. J. Cell Biol. 85: 175-186.

Forman, D. S., A. L. Padjen, and G. R. Siggins (1977) Axonal transport of organelles visualized by light microscopy: Cinemicrographic and computer analysis. Brain Res. 136: 197-213.

Gainer, H., and D. J. Fink (1982) Evidence for slow retrograde transport of serum albumin in rat sciatic nerve. Brain Res. 233: 404-408.

Gold, B. G. J. W. Griffin, and B. D. Trapp (1984) Lack of evidence for slow retrograde transport in nerve using the Bolton Hunter reagent. Soc. Neurosci. Abstr. 10: 353.

Hedreen, J. C., and G. C. Holm (1981) Retrograde and anterograde axonal transport demonstrated by intracerebral injection of a labeled protein acylating agent. Brain Res. Bull. 7: 665-670.

Hoffman, P. N., and R. J. Lasek (1975) The slow component of axonal transport. J. Cell Biol. 66: 351-366.

Katz, M. J., R. J. Lasek, P. Osbody, J. R. Whittaker, and A. I. Kaplan (1982) Bolton-Hunter reagent as a vital stain for developing systems. Devel. Biol. 90: 419-429

Laemmli, U. K. (1970) Cleavage of structural protein during the assembly of the head of bacteriophage. Nature 227: 680-685.

Lasek, R. J., J. A. Garner, and S. T. Brady (1984) Axonal transport of the cytoplasmic matrix. J. Cell Biol. 99: 2125-2215.

LaVail, J. H., and M. M. LaVail (1974) The retrograde intraaxonal transport of horseradish peroxidase in the chick visual system: A light and electron microscopic study. J. Comp. Neurol. 157: 303-358.

LaVail, J. H., S. Rapisardi, and I. K. Sugino (1980) Evidence against the smooth endoplasmic reticulum as a continuous channel for the retrograde axonal transport of horseradish peroxidase. Brain Res. 191: 3-20.

Lorenz, T., and M. Willard (1978) Subcellular fractionation of intraaxonally transported polypeptides in the rabbit visual system. Proc. Nat1. Acad. Sci. U. S. A. 75: 505-509.

Schonbach, J., Ch. Schonbach, and M. Cuenod (1971) Rapid phase of axoplasmic flow and synaptic proteins: An electron microscopical autoradiographic study. J. Comp. Neurol. 141: 485-498.

Schonbach, J., Ch. Schonbach, and M. Cuenod (1973) Distribution of transported proteins in the slow phase of axoplasmic flow. An electron microscopical autoradiographic study. J. Comp. Neurol. 152: 1-16.

Smith, R. S. (1980) The short term accumulation of axonally transported organelles in the region of localized lesions of single myclinatcd axons. $J$. Neurocytol. 9: 39-65.

Sparrow, J. R. (1981) Immunocytochemical localization of plasma proteins in neuronal perikarya. Brain Res. 212: 159-163.

Szaro, B. G., Y. P. Loh, and Hunt (1981) Use of ${ }^{3} \mathrm{H}$ N-SP to study retrograde transport in Xenopus optic nerve. Biophys. J. 33: 92a.

I sukita, $\mathrm{S}$., and $\mathrm{H}$. Ishikawa (1980) The movement of membranous organelles in axons. Electron microscopic identification of anterogradely and retrogradely transported organelles. J. Cell Biol. 84: 513-530.

Whur, P., A. Herscovics, and C. P. Leblond (1969) Radioautographic visualization of the incorporation of galactose ${ }^{3} \mathrm{H}$ and manose ${ }^{3} \mathrm{H}$ by rat thyroids in vitro in relation to the stages of thyroglobulin synthesis. J. Cell Biol. 43: 289-311.

Williams, L. R., and B. W. Agranoff (1983) Retrograde transport of goldfish optic nerve proteins labeled by $\mathrm{N}$-succinimidyl ${ }^{3} \mathrm{H}$ propionate. Brain Res. 259: 207-216. 